\title{
Comparison of ongoing COVID-19 pandemic confirmed cases/deaths weekly forecasts on continental basis using R statistical models
}

\author{
Zeydin Pala ${ }^{1, *}$, Ahmet Faruk PALA ${ }^{2}$ \\ ${ }^{1}$ Muş Alparslan University, Engineering Faculty, Department of Software Eng., Muş. Turkey, https://orcid.org/0000-0002-2642-7788 \\ 2 İnönü University, Engineering Faculty, Department of Computer Engineering, Malatya, Turkey, https://orcid.org/ 0000-0001-5841-1943
}

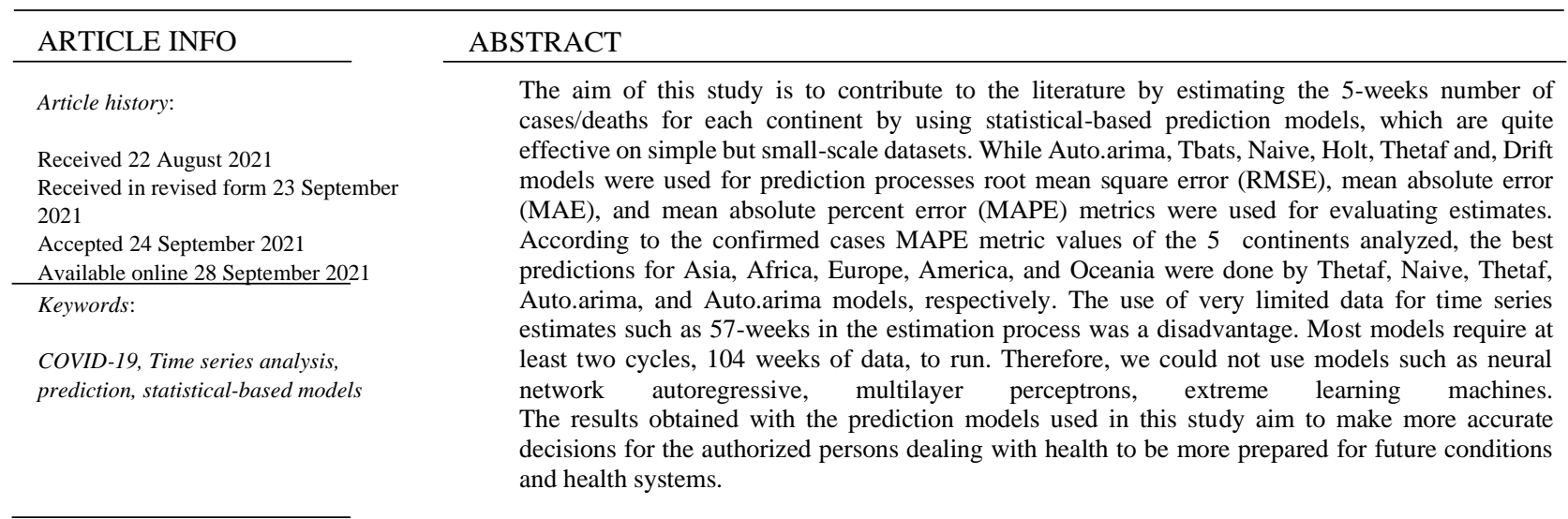

Doi: 10.24012/dumf.1002160

* Corresponding author

\section{Introduction}

As the COVID-19 virus poses a threat to people living around the world, it is very important to predict the weekly numbers of future cases/deaths to support disease prevention and help prepare health services. Although some restrictions, such as forcing people to stay at home from time to time due to the pandemic process, make life boring, it has also contributed to the cleanliness of many living spaces, especially the environment, and the easier movement of animals. The history of humanity has witnessed many epidemics that have affected the people of the world from the past to the present on a global basis. These types of epidemics have emerged in history under different names. [1]. Tyrrell and Bynoe first mentioned a Coronavirus family in their study in 1966 [2]. In the literature, corona viruses have generally been defined as viruses with large RNA with an envelope, positive single strand, affecting both humans and a wide variety of animals [3]-[6]. The COVID-19 virus, which is current on a global basis today, first appeared in Wuhan, China's Hubei province, provisionally called 2019-nCoV [7], with the spread of an unknown pneumonia epidemic in December 2019 [8]-[13].
On March 11, 2020, World Health Organization (WHO) declared the COVID-19 outbreak as an official pandemic due to the global spread and severity of the disease [14]. The COVID-19 outbreak is the most important global crisis since the Second World War. All countries of the world were affected by this crisis and continue to be affected [15]. The COVID-19 virus has a higher transmission rate than seasonal Influenza and many other diseases. In symptomatic patients, clinical signs of the disease, consisting of fever, cough, nasal congestion, fatigue and other signs of upper respiratory tract infection, usually begin less than a week later [12], [16]-[21]. COVID-19 virus not only affects people physically, but also negatively affects people's quality of life and mental health. Many factors such as prolonged quarantine and social distancing can make people depressed and cause anxiety disorders [22]. In addition, people with suspected COVID-19 transmission may experience stress, depression, nervousness, insomnia during their quarantine. People with mental health problems are affected by the emotional reactions brought about by the epidemic, and they can cause mental health to deteriorate due to higher sensitivity to stress and depression compared to other people [23]. As the 
COVID-19 pandemic has various effects on people, it has also caused a great change in many areas such as industry, sports, economic, financial and education [24]. In addition, the current economic crisis and the stagnation at commercial points caused effects in different areas. Social distancing, self-isolation and travel restrictions have resulted in a decrease in the workforce and loss of many jobs in the general economic sectors. On the other hand, the need for medical supplies and food increased significantly[12],[24]. Artificial intelligence (AI)[25], machine learning (ML) [26], [27], engineering techniques [28], and time-series methods [29]-[31] have recently made significant advances in healthcare. AI can use advanced algorithms to learn features from a vast amount of health data, and then use the information gained to assist clinical practice [32].

To our knowledge, there are several studies [33]-[38] in the literature that use different models to predict COVID-19 data, but this is the first study to use weekly continent-based confirmed data between the first week of January 2020 and the third week of January 2021.

This article contributes to the literature on weekly timeseries forecasting the COVID-19 outbreak in several ways: (i) modeling weekly data mathematically in the form of time series; (ii) to analyze weekly continental data between the first week of January 2020 and the third week of January 2021; (iii) to make separate estimates for cases and deaths by using six different statistical-based models for weekly time series on a continent basis; (iv) evaluating the forecast results with the help of RMSE, MAE, and MAPE metrics.

\section{Research Method}

\section{Study Design}

In this section, obtaining the data, modeling the data as a time series, used prediction models and performance metrics are given respectively. All of the weekly datasets used in this study, given based on the world countries COVID-19 data, were provided from the European Union open data portal site (https://data.europa.eu/euodp/data/dataset). The data used here consists of 57 weeks and starts from the first week of January 2020 and ends in the third week of January 2021. The weekly data used in the study were made available on the basis of country data in Asia, Africa, America (North continents. Weekly data have been obtained separately by taking into account the numbers of confirmed cases and deaths in countries on five continents. The graphs of cases and deaths belonging to the Asia-Africa, EuropeanAmerica and Oceania continents are shown in Figures 1, 2, and 3 , respectively, as a 57-week time-series. In addition, statistical values such as min, median, mean and max belonging to five continents are given in Table 1. As seen in Table 1, on average, the highest numbers of confirmed cases/deaths are seen in the continents of America, Europe, Asia, Africa and Oceania respectively.

As seen in Figure 1, weekly data consisting of 57 weeks on the time axis are formed between the first week of January 2020 and the third week of January 2021. The highest number of confirmed cases and deaths in Asia continent was realized as 851577 and 12466, respectively, in the second week (38th week) of September 2020. The number of confirmed cases in the Asia continent has increased since the 1st week of 2020 and reached a peak in the 38th week. It then entered a downward trend. Although the deaths numbers of the same continent increased similar to the cases trend, there were occasional decreases. As can be seen in the lower part of the same graph, the highest number of cases and deaths in the African continent was realized as 227221 and 6501, respectively, in the 1 st and 3rd week of January 2021 (i.e., the 54th and 56th weeks). In the African continent, the number of cases reached the first peak in the 29 th week and the second peak in the 56th week. The 57week chart of deaths in the African continent is quite similar to the cases chart. As seen in Figure 2, the highest number of cases in Europe continent was realized as 2092934 in the 1st week of November 2020 (i.e., at the 45th week), while the number of deaths was 39861 in the 3rd week of January 2021 (i.e., at the 56th week).

When the 57-week trend of the European continent is evaluated, it is seen that the first 3 weeks continue at the 0 level, the following weeks have risen and reached the peak in the 45th week, and then declined. On the deaths chart of the European continent, it is seen that the first peak was reached in the 15th week, followed by a downward trend and then increased and peaked again in the 56th week.

In the continent of America, located at the bottom of Figure 2 , the highest number of confirmed cases and deaths was

Table 1. Statistical distribution of 57 weeks of data used for Asia, Africa, Europe, America, and Oceania continents

\begin{tabular}{lllllllll}
\hline Continent & & \multicolumn{3}{c}{ Confirmed cases } & \multicolumn{3}{c}{ Confirmed deaths } \\
& Min & Median & Mean & Max & Min & Median & Mean & Max \\
\hline Asia & 1 & 425608 & 350950 & 851577 & 0 & 6640 & 5930 & 12466 \\
Africa & 0 & 55870 & 62804 & 227221 & 0 & 1518 & 1603 & 6501 \\
Europa & 0 & 221408 & 588342 & 2092934 & 0 & 5159 & 12996 & 39861 \\
America & 0 & 768081 & 811763 & 2699838 & 0 & 20057 & 18684 & 48277 \\
Ocenia & 0 & 686 & 1010 & 3607 & 0 & 9 & 21.18 & 148 \\
\hline
\end{tabular}

America and South America), Europe and Oceania realized as 2699838 and 48277, respectively, in the 1st and 
3rd week of January 2021 (i.e., the 54th and 56th weeks). In general, it is seen that the number of cases and deaths in the American continent increased, reaching a peak in the 54th and 56th weeks, respectively.
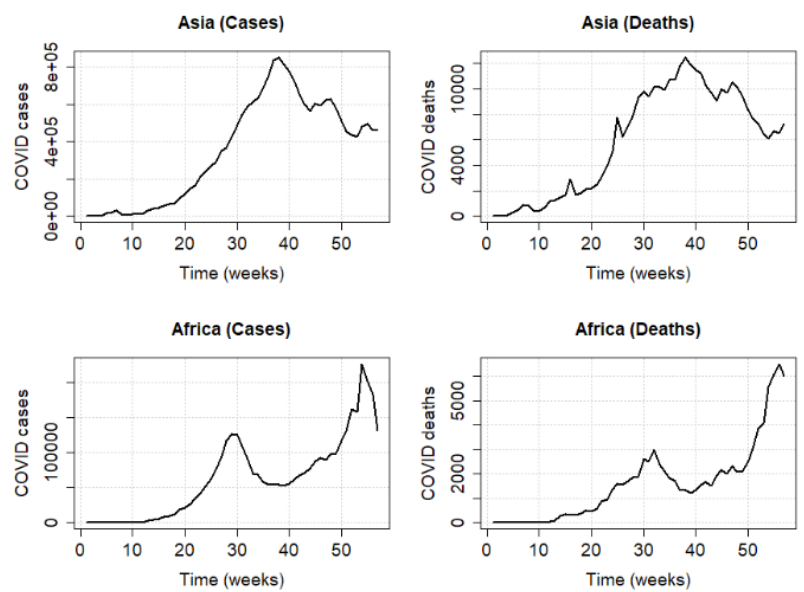

Figure 1. The confirmed cases/deaths values between the first week of 2020 and the third week of 2021 of the 57-

week time series of Asia and African continents

As seen in Figure 3, the highest number of cases in Oceania continent was 3607 in the last week of July 2020 (i.e., in the 31 st week), while the number of deaths was 39861 in the first week of September 2020 (i.e. week 36). The numbers of COVID-19 confirmed cases/deaths in Oceania continent vary considerably. The death rate in the same continent is only $2.1 \%$ of the cases rate.
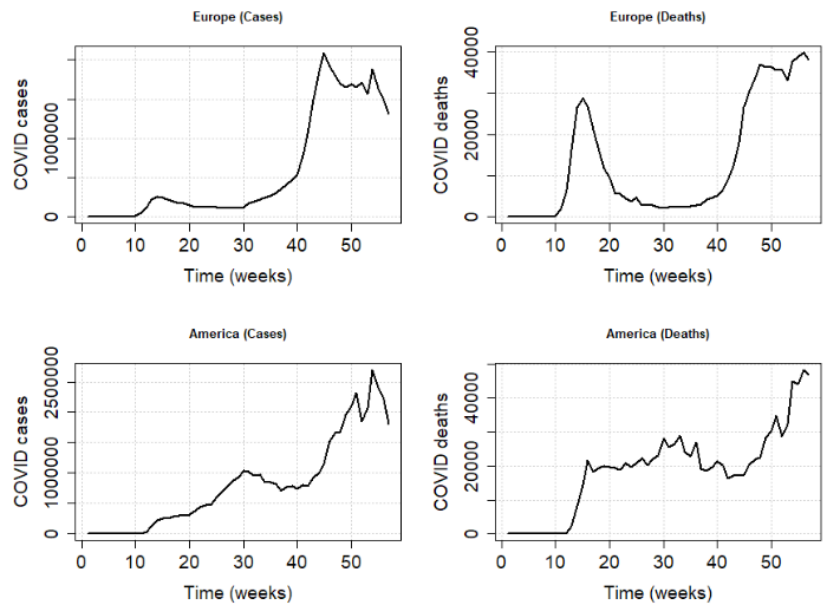

Figure 2. The confirmed cases/deaths values between the first week of 2020 and the third week of 2021 of the 57week time series belonging to the European and American continents
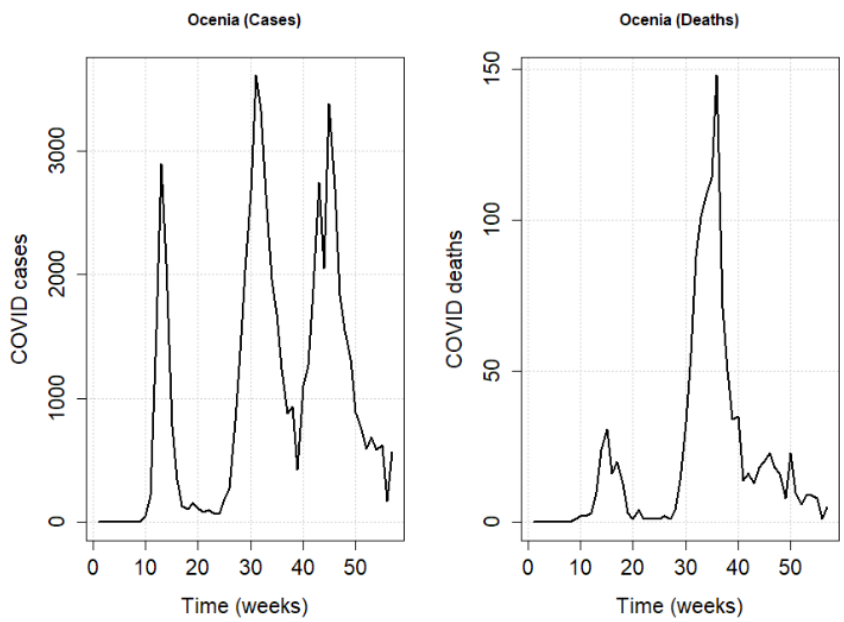

Figure 3 . The confirmed cases/deaths values between the first week of 2020 and the third week of 2021 of the 57week time series belonging to the Oceania continent

\section{Modeling EU-ODP data as time series and prediction models}

Time series analysis is commonly used in many fields due to the significant relation between the past and the future. Time series models are useful for modeling data obtained and indexed by time [36]. Time series are used for future predictions in many areas such as health [39], [40], sensor data analysis [41], speech recognition [42], economics [43], radiation forecasting [44], [45], sunspots [46], [47].

Data supplied from the EU-ODP portal can be modeled in time series format, by means of predictor variables, as follows:

$E U \_O D P=f$ (confirmed_cases /

deaths, time_of_week, error)

Note that on the right, the "error" word allows for random variation. Accordingly, the time series prediction equation will be:

$E U_{-} O D P_{n+1}=$

$f\left(E U_{O D P_{n}}, E U_{O D P_{n-1}}, E U_{O D P_{n-2}}, E U_{O D P_{n-3}}, \ldots, \varepsilon\right)$

Where subscript $\mathrm{n}$ is the present week, subscript $\mathrm{n}+1$ is the next week, subscript n-1 is the previous week, subscript n2 is the two weeks ago, subscript n-3 is the three weeks ago, and so on. $\varepsilon$ represents the random variation and the effects of variables not included in the model. Here, future forecasting is based on a variable's previous values, but not on external factors that may influence the system. In the models given hereafter, the numbers of cases / deaths obtained weekly at time $\mathrm{n}$ are shown with $y_{n}$.

In this study, the Naive method, which makes predictions based on the last observation, Drift method for all changes that occur over time, Auto Regressive Integrated Moving Average (auto.arima) method, Exponential smoothing state space model with Box-Cox (Tbats) method, Holt-Winters 
[48] method and Theta method forecast (Thetaf) method in Forecast Package imported into the $\mathrm{R}$ programming environment were used in the prediction processes. [49][51].

\section{Performance evaluation}

The consistency of EU_ODP confirmed cases/deaths data will significantly influence the prediction results in terms of calculation precision, missed values, corrupted data, and data entry errors. In time series forecasting, where the sample size is limited, data accuracy is particularly important. Preprocessing the data is one of the crucial steps of data analysis, one of the preliminary steps in that includes feature scaling. The number of weekly EU_ODP cases/deaths, y, was normalized by unit length normalization (ULN), as defined by equation (3). ULN transforms $\mathrm{x}$ to $\mathrm{x}^{\prime}$ by dividing each value of the feature vector by Euclidean length of the vector.

$\mathrm{x}^{\prime}=\frac{\mathrm{x}}{\|\mathrm{x}\|}=\frac{x}{\sqrt{\sum x^{2}}}$

Where $\mathrm{x}^{\prime}, \mathrm{x}$, and $\|\mathrm{x}\|$ is normalised value, original value and Ecludian distance vector respectively. In time series forecasting, as in the case of cross-sectional results, we first split the data into a training cycle and a test period in order to prevent overfitting and be able to measure the model's predictive output on new data.

Various statistical tests, including root mean square error (RMSE), mean absolute error (MAE), and mean absolute percent error (MAPE) were determined to analyze the performance of models using numerical time-series values. In equations 4, 5 and 6, respectively, equations for RMSE, MAE, and MAPE metrics are given.

$$
\begin{aligned}
& \text { RMSE }=\sqrt{\frac{1}{N} \sum_{n=1}^{N}\left(e_{n+h}\right)^{2}} \\
& M A E=\frac{1}{N} \sum_{n=1}^{N}\left|e_{n+h}\right| \\
& M A P E=\frac{1}{N} \sum_{n=1}^{N}\left|\frac{e_{n}}{y_{n}}\right| x 100
\end{aligned}
$$

The $\mathrm{n}, \mathrm{N}, e_{n}, y_{n}$, and $\mathrm{h}$ given in the equations show time, number of observations, prediction error, actual value and future forecast horizon, respectively.The forecast error for time period $\mathrm{n}$, denoted $e_{n}$, is defined as the difference between the actual value, $\boldsymbol{y}_{\boldsymbol{n}}$ and the forecast value, $\widehat{\boldsymbol{y}}_{\boldsymbol{n}}$ at time $n$.

$e_{n}=\boldsymbol{y}_{\boldsymbol{n}}-\widehat{\boldsymbol{y}}_{\boldsymbol{n}}$

Here, $e_{\boldsymbol{n}}$ does not mean a misunderstanding, it means an unforeseeable aspect of the observation. It can be written down as

$e_{T+\boldsymbol{h}}=\boldsymbol{y}_{N+\boldsymbol{h}}-\widehat{\boldsymbol{y}}_{N+\boldsymbol{h}} \mid N$,

where the training data is given by $\left\{y_{1}, y_{2}, y_{3}, \ldots, y_{N}\right\}$ and the test data is given by $\left\{y_{N+1}, y_{N+2}, y_{N+3}, \cdots\right\}$. Note that prediction errors vary in two ways from residuals. Firstly, the residues are measured on the training set while the predicted errors are calculated on the test set. Secondly, residues rely on one-step predictions, while forecast errors will require multi-stage predictions.

\section{Results and Discussion}

In this study, time series were created using 57-week COVID-19 confirmed cases and deaths data from Asia, Africa, Europe, America and Ocean continents. Separate time series are used for the estimation of the numbers of cases and deaths of the continents. Six different models, namely Thetaf, Drift, Auto.arima, Tbats, Naïve and Holt, were used in the estimation process of the time series. The time series for training and testing of each model is divided by $91 \%$ and $9 \%$, respectively.

57-week COVID-19 cases and deaths prediction charts of Asia, Africa, Europe, America and Ocean continents are given between Figure 4 and Figure 13 with support for six different models. In addition, the 5-week COVID-19 confirmed cases / deaths prediction test values of Asia, Africa, Europe, America and Ocean continents are given as RMSE, MAE and MAPE metric values in Table 2. When the metric values given in Table 2 for the Asia continent are evaluated together with Figures 4 and 5, it is seen that the best estimation process for both cases and deaths time series is made by Thetaf model when MAPE metric is taken as reference in 5-week estimation processes. Here, the MAPE metric value of 4.166 means that the same model made an error of $4.166 \%$ on the test data. On the other hand, the performance percentage of the model for the test data is $95.834 \%$. As seen in Table 2, model achievements on the 5week time series used for cases for Asia continent are Thetaf, Drift, Auto.arima, Tbats, Naïve and Holt. This ranking occurs as Tbats, Auto.arima, Naïve, Holt, Thetaf, and Drift for deaths time series. The Thetaf model made $11.93 \%$ less error in the prediction operations of the Asia continent based on the MAPE value on the cases data, compared to the predicted values obtained for deaths of the same continent. In other words, the performance of the predictions made on the cases data is $11.93 \%$ better than the performance on deaths data. As seen in Figures 4 and 5, blue lines display predictions for the next 5-weeks. The dark shaded area displays $80 \%$ predicted intervals. In other words, each potential value is predicted to lie in the dark shaded area with a probability of $80 \%$.

Prediction graphs of COVID-19 cases and deaths belonging to the African continent are given in Figures 6 and 7 , respectively. We see that the Drift method shows the best performance in the prediction of cases, whereas the Tbats model performs better in predicting deaths. Forecast differences in time series depend on both the model, dataset 
Table 2. Five-week confirmed cases /deaths RMSE, MAE, and MAPE metric values for the continents using six prediction models

\begin{tabular}{|c|c|c|c|c|c|c|c|}
\hline \multirow[t]{2}{*}{ Continent } & \multirow[t]{2}{*}{ Models } & \multicolumn{3}{|c|}{ COVID confirmed cases test metrics } & \multicolumn{3}{|c|}{$\begin{array}{l}\text { COVID confirmed deaths test } \\
\text { metrics }\end{array}$} \\
\hline & & RMSE & MAE & MAPE & RMSE & MAE & MAPE \\
\hline \multirow[t]{6}{*}{ Asia } & Thetaf & 0.006 & 0.005 & 4.166 & 0.019 & 0.019 & 16.096 \\
\hline & Drift & 0.006 & 0.005 & 4.198 & 0.020 & 0.019 & 16.732 \\
\hline & Auto.arima & 0.021 & 0.019 & 13.849 & 0.013 & 0.012 & 10.370 \\
\hline & Tbats & 0.025 & 0.023 & 16.455 & 0.010 & 0.009 & 7.562 \\
\hline & Naive & 0.034 & 0.031 & 22.584 & 0.013 & 0.012 & 10.371 \\
\hline & Holt & 0.034 & 0.031 & 22.611 & 0.016 & 0.013 & 11.020 \\
\hline \multirow[t]{6}{*}{ Africa } & Thetaf & 0.057 & 0.049 & 17.098 & 0.010 & 0.100 & 28.572 \\
\hline & Drift & 0.056 & 0.047 & 17.051 & 0.102 & 0.092 & 26.221 \\
\hline & Auto.arima & 0.078 & 0.062 & 25.873 & 0.068 & 0.061 & 17.558 \\
\hline & Tbats & 0.182 & 0.136 & 56.807 & 0.041 & 0.036 & 10.641 \\
\hline & Naive & 0.057 & 0.045 & 16.930 & 0.116 & 0.105 & 30.060 \\
\hline & Holt & 0.057 & 0.048 & 17.076 & 0.100 & 0.090 & 25.853 \\
\hline \multirow[t]{6}{*}{ Europe } & Thetaf & 0.040 & 0.035 & 16.546 & 0.016 & 0.015 & 6.014 \\
\hline & Drift & 0.047 & 0.040 & 19.116 & 0.012 & 0.010 & 4.073 \\
\hline & Auto.arima & 0.044 & 0.038 & 18.035 & 0.016 & 0.015 & 6.049 \\
\hline & Tbats & 0.041 & 0.036 & 17.031 & 0.013 & 0.012 & 4.871 \\
\hline & Naive & 0.046 & 0.040 & 18.865 & 0.021 & 0.020 & 7.853 \\
\hline & Holt & 0.047 & 0.040 & 19.320 & 0.021 & 0.020 & 7.854 \\
\hline \multirow[t]{6}{*}{ America } & Thetaf & 0.055 & 0.046 & 16.214 & 0.087 & 0.081 & 30.768 \\
\hline & Drift & 0.052 & 0.044 & 10.480 & 0.082 & 0.076 & 28.869 \\
\hline & Auto.arima & 0.052 & 0.044 & 10.480 & 0.092 & 0.086 & 32.601 \\
\hline & Tbats & 0.059 & 0.051 & 18.163 & 0.099 & 0.093 & 35.335 \\
\hline & Naive & 0.052 & 0.044 & 15.672 & 0.092 & 0.086 & 32.600 \\
\hline & Holt & 0.052 & 0.044 & 10.486 & 0.053 & 0.048 & 17.906 \\
\hline \multirow[t]{6}{*}{ Ocenia } & Thetaf & 0.021 & 0.013 & 67.227 & 0.012 & 0.010 & 155.190 \\
\hline & Drift & 0.019 & 0.012 & 61.629 & 0.010 & 0.009 & 130.748 \\
\hline & Auto.arima & 0.017 & 0.010 & 54.625 & 0.010 & 0.009 & 122.333 \\
\hline & Tbats & 0.020 & 0.012 & 62.310 & 0.010 & 0.009 & 118.540 \\
\hline & Naive & 0.018 & 0.010 & 55.805 & 0.014 & 0.011 & 181.966 \\
\hline & Holt & 0.019 & 0.011 & 60.344 & 0.010 & 0.009 & 117.734 \\
\hline
\end{tabular}

length, and trend/seasonality effects in the data. Therefore, it is possible that different models make better predictions on different datasets. The absence of cases in the African continent in the first 8 weeks of 2020 and the absence of deaths in the first 9 weeks caused a decrease in performance in estimation procedures. Therefore, it is seen in Table 2 that the predicted values of the African continent are less accurate than the Asian continent, considering the MAPE metric.

The estimation charts of COVID-19 cases and deaths belonging to the European continent are given in Figures 8 and 9 , respectively, for 5-weeks, and the estimation metric values are given in Table 2.

Accordingly, we see that the Thetaf model stands out with $16.545 \%$ MAPE value in the cases estimates and the Drift model with $4.073 \%$ MAPE value in deaths estimates. 5week COVID-19 cases and deaths prediction charts for the
Americas are given in Figures 10 and 11, respectively, and metric values are given in Table 2. In the Cases estimates, we see that Drift, Auto.arima and Holt models have 10.480, 10.480 and 10.486 MAPE values, respectively. The rates of error and correct prediction of these models are quite close to each other. However, in the deaths predictions, we see that the Holt model predicts with an error margin of $17.906 \%$, that is, it makes $82.094 \%$ correct predictions.

For the Ocenia continent, 5-week COVID-19 cases and deaths prediction charts are given in Figures 12 and 13, respectively, and metric values are given in Table 2 . For the Ocenia continent cases predictions, the auto.arima model provided the best performance with $54.625 \%$ error, while the deaths predictions provided holt with $117.734 \%$ error.

Due to the irregular number of cases and deaths in Ocenia continent, the performance of the models in the prediction process was very low. For the five continents whose 
performances were evaluated, the lowest performance was obtained in the cases and deaths estimation processes of Ocenia continent. The irregularity of the data in the time series negatively affected the performance of the model.
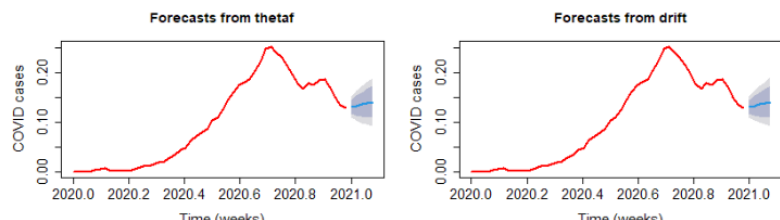

Time (weeks)
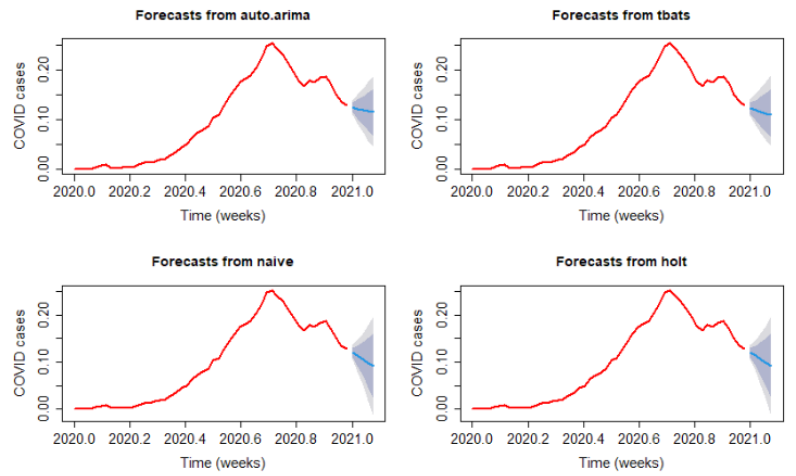

Figure 4. Representation of 5-week estimates with $80 \%$ and $95 \%$ prediction intervals using six different statistical models of cases in the Asian continent.
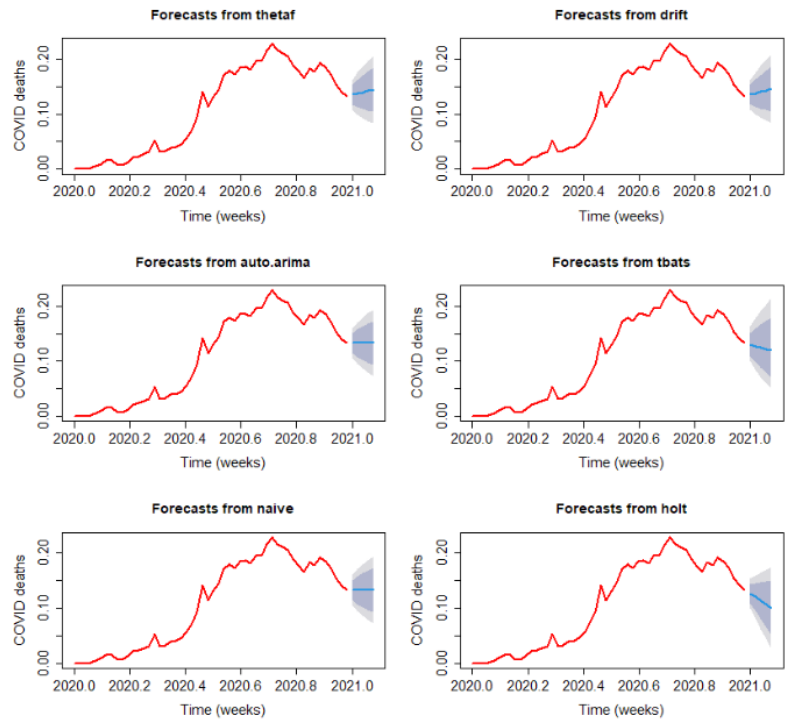

Figure 5. Representation of 5-week estimates with $80 \%$ and $95 \%$ prediction intervals using six different statistical models of deaths in the Asian continent.
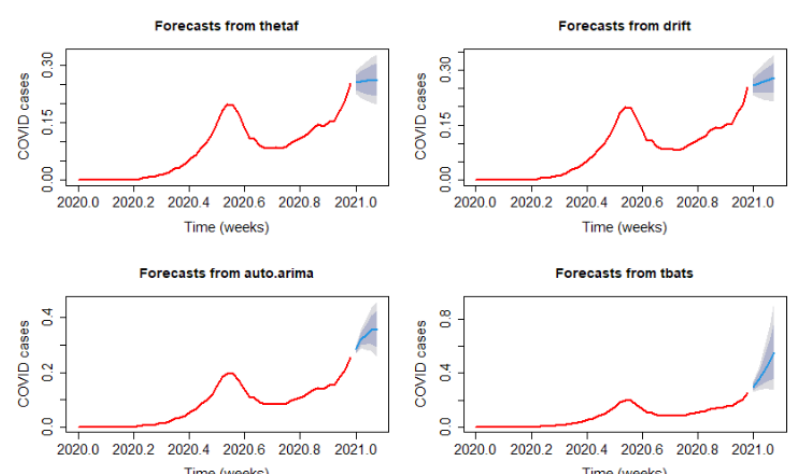

Time (weeks)

Time (weeks)
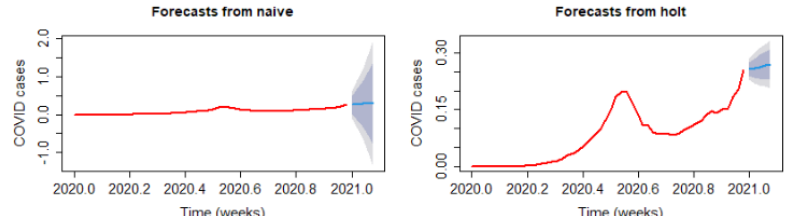

Time (weeks)

Figure 6. Representation of 5-week estimates with $80 \%$ and $95 \%$ prediction intervals using six different statistical models of cases in the Africa continent.
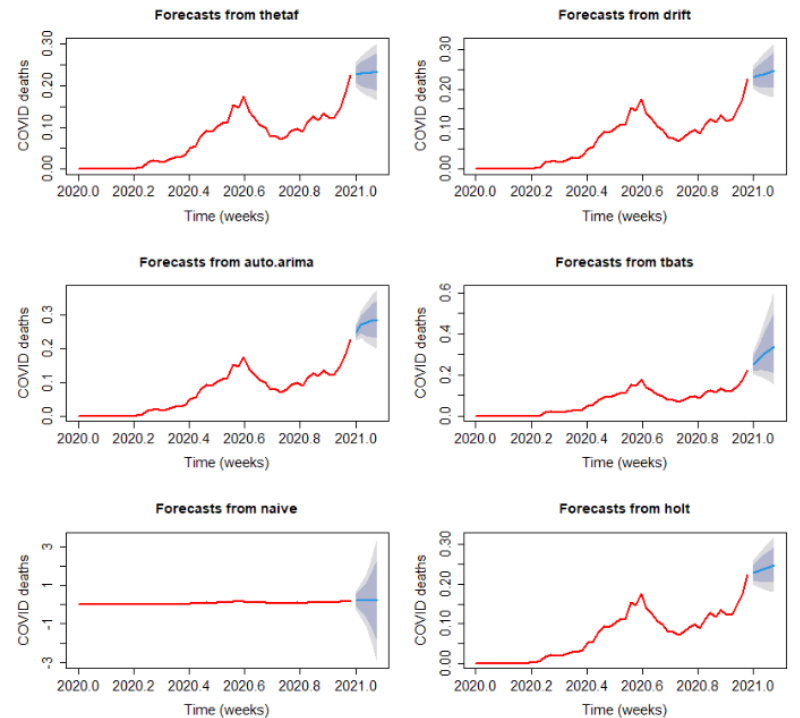

Figure 7. Representation of 5-week estimates with $80 \%$ and $95 \%$ prediction intervals using six different statistical models of deaths in the Asian continent. 

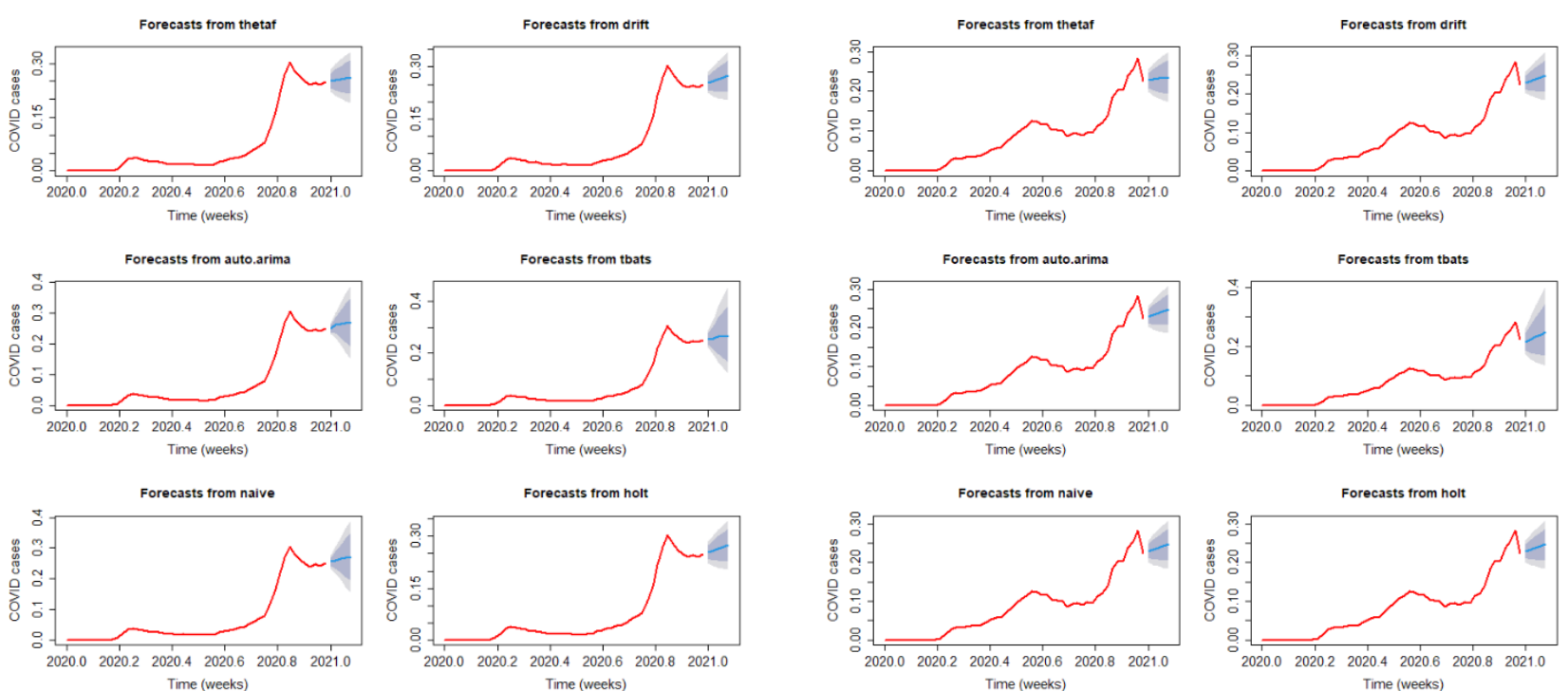

Figure 8. Representation of 5-week estimates with $80 \%$ and $95 \%$ prediction intervals using six different statistical models of cases in the European continent.
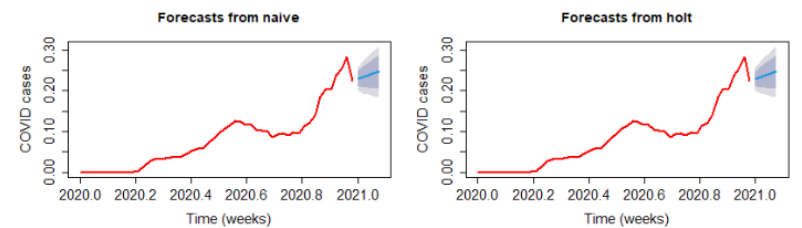

Figure 10. Representation of 5-week estimates with $80 \%$ and $95 \%$ prediction intervals using six different statistical models of cases in the American continent.
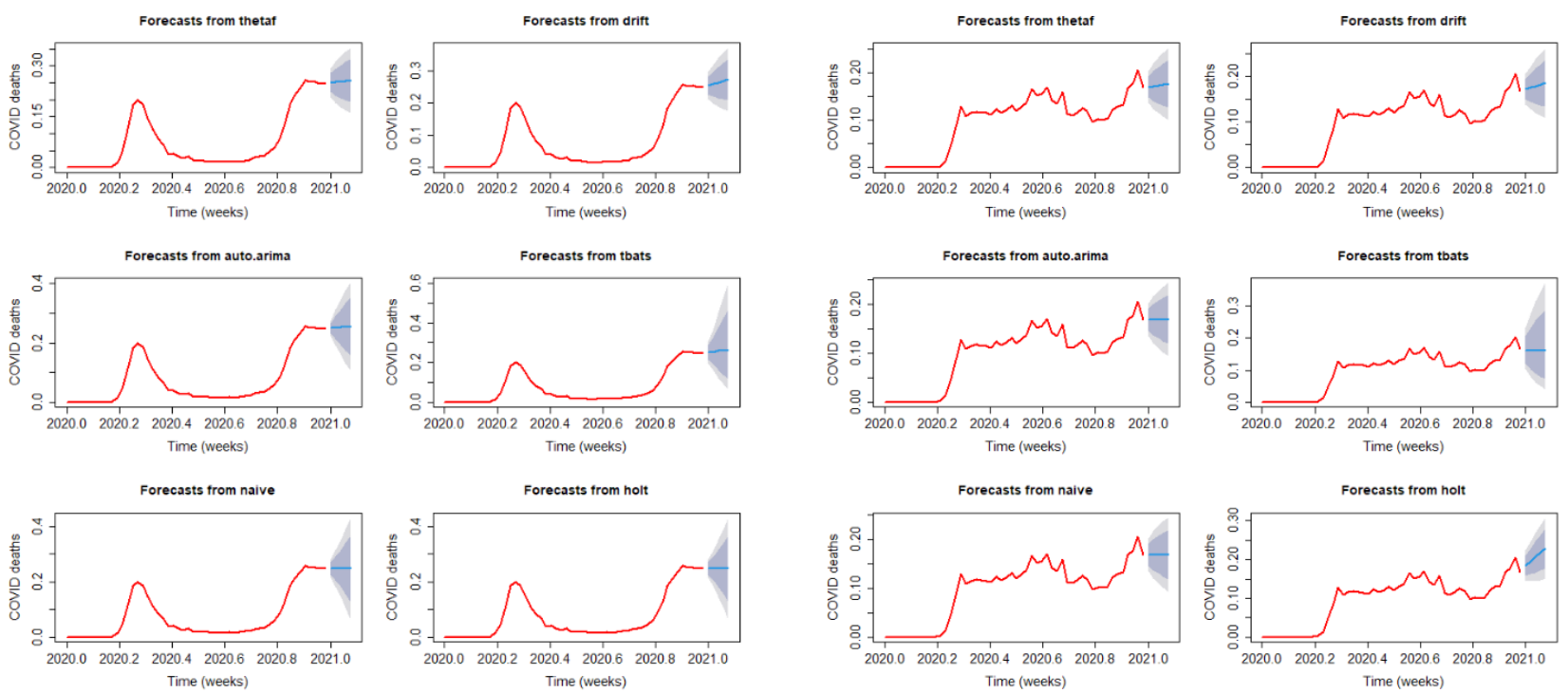

Figure 9. Representation of 5-week estimates with $80 \%$ and $95 \%$ prediction intervals using six different statistical models of deaths in the European continent.
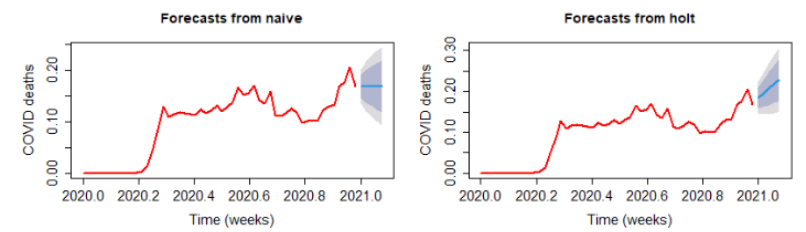

Figure 11. Representation of 5-week estimates with $80 \%$ and $95 \%$ prediction intervals using six different statistical models of deaths in the American continent. 

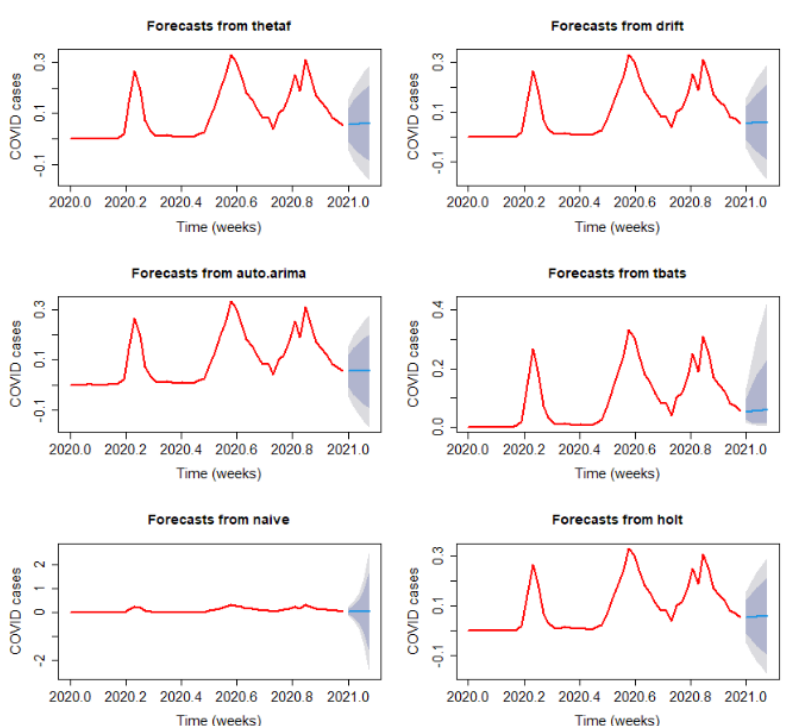

Figure 12. Representation of 5-week estimates with $80 \%$ and $95 \%$ prediction intervals using six different statistical models of cases in the Oceania continent.
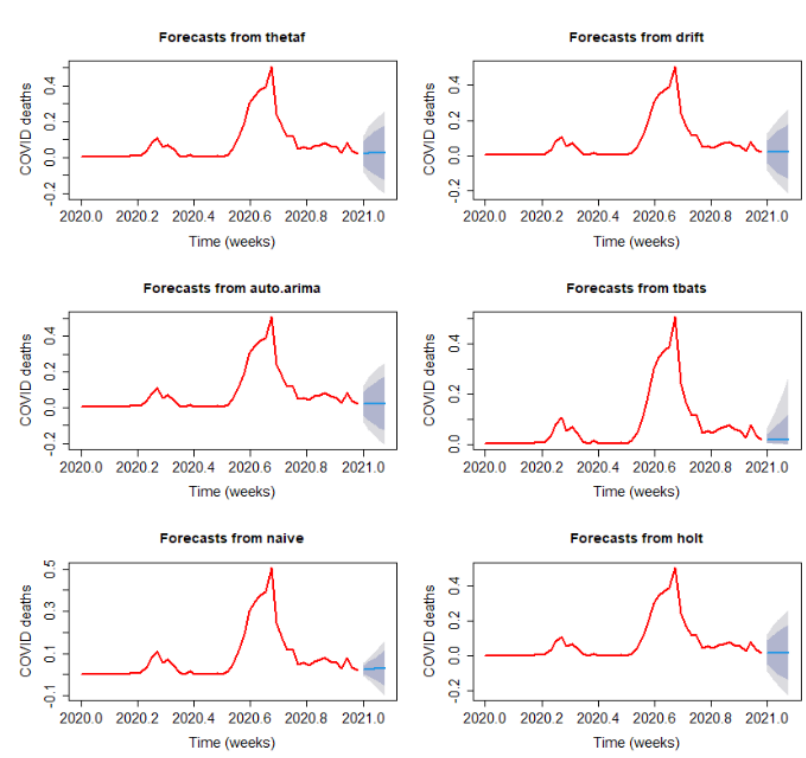

Figure 13. Representation of 5-week estimates with $80 \%$ and $95 \%$ prediction intervals using six different statistical models of deaths in the Oceania continent.

\section{Conclusion}

In this study, R-based statistical models, some of which have not been used in the forecasting of limited time series until now, are used and their performances are evaluated. In other words, the authors evaluated the usability of weekly future predictions of COVID-19, which continues to be effective globally, with time series models. Six different models such as Naive, Drift, auto.arima, Tbats, HoltWinters, and Thetaf method were used for prediction operations. RMSE, MAE, and MAPE metrics were used to evaluate the performance of the models used for 5-week predictions. The results obtained were evaluated on the basis of continents.

The accuracy of traditional estimation largely depends on the availability of the data [52]. The fact that the data used in the estimates are limited affects the estimation accuracy very negatively.

According to the confirmed cases MAPE metric values of the five continents analyzed, the best predictions for Asia, Africa, Europe, America, and Oceania were done by Thetaf, Naive, Thetaf, Auto.arima, and Auto.arima models, respectively. The use of very limited data for time series estimates such as 57-weeks in the estimation process was a disadvantage. Most models require at least two cycles, 104weeks of data, to run. Therefore, we could not use models such as Neural network autoregressive, Multilayer Perceptrons, Extreme learning machines. Especially since we had to make predictions using a limited data set such as 57-weeks, we could not reach the desired levels in model performances. In a sense, more data means better predictions for time series.

The socio-economic, demographic, and environmental conditions of the countries in the continents where data are collected differ. In addition, there is a serious shortage of nurses in low and medium-developing countries. In comparison, the number of hospital beds per thousand people and access to health care services in these less developed countries is still very limited. However, wealthier and relatively more developed countries have more overweight people relative to lower GDP countries and are thus more vulnerable to COVID-19 [26].

\section{Ethical approval}

No ethical approval was required for this study. The analysis uses only publicly available data reported in the European Union open data portal site.

\section{References}

[1] S. D. Pitlik, "Covid-19 compared to other pandemic diseases," Rambam Maimonides Med. J., vol. 11, no. 3, pp. 1-17, 2020.

[2] D. A. Tyrrell and M. L. Bynoe, "Cultivation of viruses from a high proportion of patients with colds.," Lancet, vol. 1, no. 7428, pp. 76-77, 1966.

[3] N. Zhu et al., "A Novel Coronavirus from Patients with Pneumonia in China, 2019," N. Engl. J. Med., vol. 382, no. 8, pp. 727-733, 2020. 
[4] J. Cui, F. Li, and Z. L. Shi, "Origin and evolution of pathogenic coronaviruses," Nat. Rev. Microbiol., vol. 17, no. 3, pp. 181-192, 2019.

[5] R. Wölfel et al., "Virological assessment of hospitalized patients with COVID-2019," Nature, vol. 581, no. 7809, pp. 465-469, 2020.

[6] D. Sornette, E. Mearns, M. Schatz, K. Wu, and D. Darcet, "Interpreting, analysing and modelling COVID-19 mortality data," Nonlinear Dyn., vol. 101, no. 3, pp. 1751-1776, 2020.

[7] S. Perlman, "Another Decade, Another Coronavirus," N. Engl. J. Med., vol. 382, no. 8, pp. 760-762, 2020.

[8] C. Wang, P. W. Horby, F. G. Hayden, and G. F. Gao, "A novel coronavirus outbreak of global health concern," Lancet, vol. 395, no. 10223, pp. 470-473, 2020 .

[9] Fang, "Sensitivity of Chest CT for COVID-19: Comparison to RT-PCR," Radiology, vol. 395, no. 3, pp. A1-A2, 2020.

[10] N. Chen et al., "Epidemiological and clinical characteristics of 99 cases of 2019 novel coronavirus pneumonia in Wuhan, China: a descriptive study," Lancet, vol. 395, no. 10223, pp. 507-513, 2020.

[11] W. Wang et al., "Detection of SARS-CoV-2 in Different Types of Clinical Specimens," JAMA - J. Am. Med. Assoc., vol. 323, no. 18, pp. 1843-1844, 2020.

[12] T. Singhal, "Review on COVID19 disease so far," Indian J. Pediatr., vol. 87, no. April, pp. 281-286, 2020.

[13] D. Wang et al., "Clinical Characteristics of 138 Hospitalized Patients with 2019 Novel CoronavirusInfected Pneumonia in Wuhan, China," JAMA - J. Am. Med. Assoc., vol. 323, no. 11, pp. 1061-1069, 2020.

[14] Ö. Açikgöz and A. Günay, "The early impact of the Covid-19 pandemic on the global and Turkish economy," Turkish J. Med. Sci., vol. 50, no. SI-1, pp. 520-526, 2020.

[15] S. Boccaletti, W. Ditto, G. Mindlin, and A. Atangana, "Modeling and forecasting of epidemic spreading: The case of Covid-19 and beyond," Chaos, Solitons and Fractals, vol. 135, 2020.

[16] W. Guan et al., "Clinical Characteristics of Coronavirus Disease 2019 in China," N. Engl. J. Med., vol. 382, no. 18, pp. 1708-1720, 2020.

[17] T. P. Velavan and C. G. Meyer, "The COVID-19 epidemic," Trop. Med. Int. Heal., vol. 25, no. 3, pp. 278-280, 2020.

[18] C. Huang et al., "Clinical features of patients infected with 2019 novel coronavirus in Wuhan, China," Lancet, vol. 395, no. 10223, pp. 497-506, 2020.
[19] M. L. Holshue et al., "First Case of 2019 Novel Coronavirus in the United States," N. Engl. J. Med., vol. 382, no. 10, pp. 929-936, 2020.

[20] K. Q. Kam et al., "A well infant with coronavirus disease 2019 with high viral load," Clin. Infect. Dis., vol. 71, no. 15, pp. 847-849, Aug. 2020.

[21] S. R. Weiss and J. L. Leibowitz, Coronavirus pathogenesis, 1st ed., vol. 81. Elsevier Inc., 2011.

[22] M. L. McGowan, A. H. Norris, and D. Bessett, "Care Churn - Why Keeping Clinic Doors Open Isn't Enough to Ensure Access to Abortion," $N$. Engl. J. Med., vol. 383, no. 6, pp. 508-510, 2020.

[23] H. Yao, J. H. Chen, and Y. F. Xu, "Patients with mental health disorders in the COVID-19 epidemic," The Lancet Psychiatry, vol. 7, no. 4, p. e21, 2020.

[24] M. Nicola et al., "The socio-economic implications of the coronavirus pandemic ( COVID-19): A review," Int. J. Surg., vol. 78, no. April, pp. 185193, 2020.

[25] Z. Pala and O. Özkan, "Artificial Intelligence Helps Protect Smart Homes against Thieves," DÜMF Mühendislik Derg., vol. 11, no. 3, pp. 945-952, 2020.

[26] S. S. Mohamed Ali, A. H. Alsaeedi, D. AlShammary, H. H. Alsaeedi, and H. W. Abid, "Efficient intelligent system for diagnosis pneumonia (SARSCOVID19) in X-ray images empowered with initial clustering," Indones. J. Electr. Eng. Comput. Sci., vol. 22, no. 1, pp. 241251, 2021.

[27] Z. Pala and M. Şana, "Attackdet: Combining web data parsing and real-time analysis with machine learning," J. Adv. Technol. Eng. Res., vol. 6, no. 1, pp. 37-45, 2020.

[28] B. Saman, M. M. A. Eid, and M. M. Eid, "Recently employed engineering techniques to reduce the spread of COVID-19 (Corona Virus disease 2019): A review study," Indones. J. Electr. Eng. Comput. Sci., vol. 22, no. 1, pp. 277-286, 2021.

[29] İ. H. Ünlük and Z. Pala, "Prediction of monthly electricity consumption used in $\mathrm{Mu}$ ş Alparslan University Complex by means of Classical and Deep Learning methods," Int. Conf. Data Sci. Mach. Learn. Stat. - 2019, vol. 1, no. 1, pp. 237-239, 2019.

[30] Z. Pala, "Using forecastHybrid Package to Ensemble Forecast Functions in the R," Int. Conf. Data Sci. Mach. Learn. Stat. - 2019, vol. 1, no. 1, pp. 45-47, 2019.

[31] E. Yaldı and Z. Pala, "Time Series Analysis of Radiological Data of Outpatients and Inpatients in Emergency Department of Mus State Hospital," Int. Conf. Data Sci. Mach. Learn. Stat. - 2019, pp. 234236, 2019.

[32] F. Jiang et al., "Artificial intelligence in healthcare: 
Past, present and future," Stroke and Vascular Neurology, vol. 2, no. 4. BMJ Publishing Group, pp. 230-243, 01-Dec-2017.

[33] S. Chakraborti et al., "Evaluating the plausible application of advanced machine learnings in exploring determinant factors of present pandemic: A case for continent specific COVID-19 analysis," Sci. Total Environ., vol. 765, p. 142723, 2020.

[34] L. Zhong, L. Mu, J. Li, J. Wang, Z. Yin, and D. Liu, "Early Prediction of the 2019 Novel Coronavirus Outbreak in the Mainland China Based on Simple Mathematical Model," IEEE Access, vol. 8, pp. 51761-51769, 2020.

[35] T. Dehesh, H. A. Mardani-Fard, and P. Dehesh, "Forecasting of COVID-19 Confirmed Cases in Different Countries with ARIMA Models," medRxiv. medRxiv, 18-Mar-2020.

[36] M. Maleki, M. R. Mahmoudi, M. H. Heydari, and K. H. Pho, "Modeling and forecasting the spread and death rate of coronavirus (COVID-19) in the world using time series models," Chaos, Solitons and Fractals, vol. 140, p. 110151, Nov. 2020.

[37] A. Zeroual, F. Harrou, A. Dairi, and Y. Sun, "Deep learning methods for forecasting COVID-19 timeSeries data: A Comparative study," Chaos, Solitons and Fractals, vol. 140, p. 110121, Nov. 2020.

[38] T. B. Alakus and I. Turkoglu, "Comparison of deep learning approaches to predict COVID-19 infection," Chaos, Solitons and Fractals, vol. 140, 2020 .

[39] J. A. Doornik, J. L. Castle, and D. F. Hendry, "Short-term forecasting of the coronavirus pandemic," Int. J. Forecast., 2020.

[40] Z. Pala and A. F. Pala, "Perform Time-series Predictions in the R Development Environment by Combining Statistical-based Models with a Decomposition-based Approach," J. Muş Alparslan Univ. Fac. Eng. Archit., vol. 1, no. 1, pp. 1-13, 2020.

[41] Y. Yang, J. Dong, X. Sun, E. Lima, Q. Mu, and X. Wang, "A CFCC-LSTM Model for Sea Surface Temperature Prediction," IEEE Geosci. Remote Sens. Lett., vol. 15, no. 2, pp. 207-211, 2018.
[42] Y. Lecun, Y. Bengio, and G. Hinton, "Deep learning," Nature, vol. 521, no. 7553, pp. 436-444, 2015.

[43] P. M. Maçaira, A. M. Tavares Thomé, F. L. Cyrino Oliveira, and A. L. Carvalho Ferrer, "Time series analysis with explanatory variables: A systematic literature review," Environmental Modelling and Software, vol. 107. Elsevier Ltd, pp. 199-209, 01Sep-2018.

[44] Z. Pala, İ. H. Ünlük, and E. Yaldız, "Forecasting of electromagnetic radiation time series: An empirical comparative approach," Appl. Comput. Electromagn. Soc. J., vol. 34, no. 8, 2019.

[45] Z. Pala, "Examining EMF Time Series Using Prediction Algorithms With R," vol. 44, no. 2, pp. 223-227, 2021.

[46] Z. Pala and R. Atici, "Forecasting Sunspot Time Series Using Deep Learning Methods," Sol. Phys., vol. 294, no. 5, 2019.

[47] R. Atıc1 and Z. Pala, "Prediction of the Ionospheric foF2 Parameter Using R Language Forecasthybrid Model Library Convenient Time," Wirel. Pers. Commun., no. doi.org/10.1007/s11277-021-09050-6 Prediction, pp. 1-20, 2021.

[48] R. Calegari, F. S. Fogliatto, F. R. Lucini, J. Neyeloff, R. S. Kuchenbecker, and B. D. Schaan, "Forecasting Daily Volume and Acuity of Patients in the Emergency Department," 2016.

[49] R. J. Hyndman and G. Athanasopoulos, Forecasting : Principles and Practice, 2nd editio. Australia: Monash University, 2018.

[50] R. J. Hyndman, A. B. Koehler, R. D. Snyder, and S Grose, "A state space framework for automatic forecasting using exponential smoothing methods," Int. J. Forecast., vol. 18, no. 3, pp. 439-454, 2002.

[51] Rob J. Hyndman and Yeasmin Khandakar, "Automatic Time Series Forecasting: The forecast Package for R," J. Stat. Softw., vol. 27, no. 3, p. 22, 2008.

[52] F. Petropoulos and S. Makridakis, "Forecasting the novel coronavirus COVID-19," PLoS One, vol. 15, no. 3, pp. 1-8, 2020. 